\title{
BMJ Open Evaluation of the intensive outpatient clinic: study protocol for a prospective study of high-cost, high-need patients in the University of Utah Health system
}

\author{
Brittany L Bannon, ${ }^{1}$ Michelle Lucier, ${ }^{1}$ Angela Fagerlin, ${ }^{1,2}$ Jaewhan Kim, ${ }^{3}$ \\ Bernadette Kiraly, ${ }^{4}$ Peter Weir, ${ }^{5}$ Elissa M Ozanne ${ }^{1}$
}

To cite: Bannon BL, Lucier M, Fagerlin A, et al. Evaluation of the intensive outpatient clinic: study protocol for a prospective study of high-cost, high-need patients in the University of Utah Health system. BMJ Open 2019;9:e024724. doi:10.1136/ bmjopen-2018-024724

\section{- Prepublication history for} this paper is available online. To view these files, please visit the journal online (http://dx.doi. org/10.1136/bmjopen-2018024724).

Received 11 June 2018 Revised 18 October 2018 Accepted 29 November 2018

\section{ABSTRACT}

Introduction The University of Utah (UofU) Health intensive outpatient clinic (IOC) is a primary care clinic for medically complex (high-cost, high-need) patients with Medicaid. The clinic consists of a multidisciplinary care team aimed at providing coordinated, comprehensive and patient-centred care. The protocol outlines the quantitative design of an evaluation study to determine the IOC's effects on reducing healthcare utilisation and costs, as well as improving patient-reported health outcomes and quality of care.

Methods and analysis High-risk patients, with high utilisation and multiple chronic illnesses, were identified in the Medicaid ACO population managed by the UofU Health plans for IOC eligibility. A prospective, case-control study design is being used to match $100 \mathrm{IOC}$ patients to 200 control patients (receiving usual care within the UofU) based on demographics, health utilisation and medical complexity for evaluating the primary outcome of change in healthcare utilisation and costs. For the secondary outcomes of patient health and care quality, a prepost design will be used to examine within-person change across the 18 months of follow-up (ie, before and after IOC intervention). Logistic regression and hierarchical, longitudinal growth modelling are the two primary modelling approaches.

Ethics and dissemination This work has received ethics approval by the UofU Institutional Review Board. Results from the evaluation of primary and secondary outcomes will be disseminated in scientific research journals and presented at national conferences.

\section{BACKGROUND}

In 2002, the top $5 \%$ of healthcare spenders within the US population accounted for $49 \%$ of the nation's total healthcare costs. In contrast, $50 \%$ of the US population with the lowest health spending represented only $3 \%$ of the nation's total healthcare costs. ${ }^{1}$ Today, the top $1 \%$ of patients still account for $>20 \%$ of US healthcare expenditures. ${ }^{2}$ Importantly, these expenditures are relatively stable from year-to-year; of the top $5 \%$ of health spenders in 2002, nearly $34 \%$ of

\section{Strengths and limitations of this study}

- A longitudinal, prepost intervention design with a wide range of robust, validated outcome measures are strengths of this study.

- The study uses a quasi-experimental design, which limits causal inferences.

- The study design does not allow identification of which specific components of the IOC care model are the most effective; rather, it focuses on the efficacy of the IOC in its entirety.

- Difficulty with patient recruitment, commonly noted in similar evaluation studies, might limit the sample size and statistical power for testing study hypotheses.

these individuals maintained their ranking through the following year. ${ }^{3}$ Furthermore, the proportion of the highest utilisers who maintain their high spending has continued to increase. ${ }^{3}$ Individuals with this pattern of healthcare spending have been referred to as 'super-utilisers', due to their high-frequency (and often preventable) use of hospital and emergency department services, as well as the high costs associated with these visits. Borrowing from the literature and our experience, a more accurate term to describe this population is 'high-risk/high-need'-a term that focuses on the patient's medical and social needs rather than their medical spending ('super-utiliser').

High-risk, high-need patients tend to be medically complex, with comorbid chronic conditions that are poorly controlled due to mental health issues, substance abuse or other psychosocial stressors (eg, food insecurity, homelessness, social disruption or lack of social support).$^{4}$ These patients are more likely to be older, female, have higher out-of-pocket healthcare expenses relative to their income, have multiple 
chronic conditions and report poorer self-rated health and physical functioning as compared with the rest of the population. ${ }^{1-5}$ Many are either uninsured or publicly insured through Medicare or Medicaid. ${ }^{56}$ These patients frequently receive their medical care in emergency departments and hospitals, which adopt traditional approaches to care that emphasise the acute, specialised treatment and diagnosis of clinical problems, rather than treating the whole patient. However, because these high-need patients face a variety of ongoing medical, behavioural and social complications, the fragmented and specialised care they receive results in both unmet needs for individual care and higher costs to the health system.

In response to this need for innovative care models to serve the highest-risk, highest-cost patients, the University of Utah (UofU) Health began the intensive outpatient clinic (IOC) in February of 2017. The IOC enrols patients who have the highest rates of healthcare utilisation in the University's Health system, and who have multiple, chronic health conditions for which they might be receiving highly fragmented care. The IOC, with an interdisciplinary care team dedicated to providing patient-centred care, will deliver comprehensive, coordinated and customised primary care to these patients. Such care will be designed to address the full continuum of health needs and care preferences within our patient population. Previous clinical models that are similar to the IOC have had success with outcomes such as reduced hospitalisations, emergency department visits and total healthcare costs, as well as improvements in patients' activation and engagement in their care-a key factor known to impact clinical outcomes in the care process. ${ }^{78}$ However, little is known about patient-reported outcomes from these care delivery models beyond activation and engagement. Therefore, this study will prospectively evaluate the IOC's effects on healthcare utilisation, costs and patient health and healthcare quality to assess the extent to which the IOC will reduce overall healthcare utilisation/costs and improve patients' disease management and overall experiences with their healthcare.

\section{OBJECTIVES}

The objectives of the proposed study are to evaluate the IOC's effect on 1) healthcare costs and utilisation and 2) patient-reported health and quality of care, to determine whether this type of clinic will improve the quality and delivery of care and reduce healthcare spending for high-utilising patients.

The proposed study aims to answer the following research questions:

1. Compared with standard care among medically complex and high-utilising UofU Health patients who are not enrolled in the IOC, will the IOC reduce healthcare spending and utilisation-including reductions in emergency department visits, hospital admissions and length of stay and total healthcare costs (ie, sum of medical reimbursed amount and prescription reimbursed amount)?

2. Compared with their first clinic visit (before receiving any care at the IOC), will the IOC patients report improvements in their health (including physical function, quality of life) and quality of care (trust in one's provider, self-efficacy, perceived access to community resources, care coordination) across 18 months of follow-up?

\section{STUDY DESIGN}

To answer the first research question, a prospective matched case-control study will be conducted for the IOC for patients in the UofU Health system in Salt Lake City, Utah, USA. The allocation ratio of cases to controls will be 1:2. Following the recommendation from Austin, ${ }^{9}$ we assume that the 1:2 matching will improve precision and minimise bias. For the evaluation of healthcare cost and utilisation, propensity score-matched control groups will be identified from the University of Utah Health Plans (UUHP) claims database. Healthcare costs and utilisation along with demographic information (such as age, gender) and medical history (eg, comorbidities, disease severity and index appointment/visit date) of the case and control groups will be from the UUHP claims database.

To answer the second research question, a prepost study design will be used to evaluate improvements in IOC patients' health and quality of care. The intervention group will be recruited from their first visit at the IOC and invited to complete four surveys prospectively for a period of 18 months.

\section{METHODS}

\section{Patient and public involvement}

The development of research questions, outcome measures and design of the study was informed by: 1) the study team's interactions with patients during clinical visits and 2) an ongoing, qualitative study of patients and providers at the clinic. These interviews have aided the study team's understanding of patients' past experiences with healthcare, priorities for their health and preferences for the receipt and delivery of care. The results of this study will be disseminated to study participants after the completion of the study, through summary results and stories provided in newsletters.

\section{Study setting}

The IOC is part of the UofU Health and began its operations in February 2017. The clinic is located in West Valley City (a suburb of Salt Lake City that provides a convenient location for IOC patients). The IOC provides patients with $24 / 7$ phone access to providers, same-day appointment scheduling, integrative care across specialties, care coordination across physical and behavioural health providers, case management and self-management education provided by licensed clinical social workers/ 
nurse practitioners/registered nurse care coordinators and medication interventions and collaborative practice care for chronic diseases performed by a clinical pharmacist (PharmD). Enrolment in the study began in July 2017 , and is ongoing.

\section{Participants/inclusion and exclusion criteria IOC patient characteristics}

There are a number of criteria potential patients need to meet in order to be eligible for care at the IOC. Patients are candidates for the IOC if they fall into the category of high-risk and high-cost, defined by a high number of comorbidities, hospitalisations and emergency department visits, as well as seeing multiple providers and accounting for a high proportion of costs to the healthcare system. Other indicators include insurance status (publiclyinsured through Medicaid), social or behavioural health concerns (eg, homelessness or food insecurity, substance abuse or mental health disorder). This pool of potential patients has their medical records reviewed by the IOC staff who refine the selection of patients to those who struggle to engage with the health system (poor compliance, high no-show rate, poorly controlled disease states). Thus, the identification of eligible IOC patients is an iterative process using both predictive modelling and provider chart review and nominations. Exclusion criteria include: 1) patients whose sole medical problem is a new-onset cancer diagnosis or an organ transplant; 2) patients with behavioural health issues that exceed the clinic's ability to assist; 3) patients receiving exclusive palliative care or those at high risk for mortality in the coming weeks and 4) patients $>65$ years of age (ie, Medicare). All patients who receive care at the IOC are invited to enrol in the study; however, for the secondary analysis, all new patients are eligible to participate in the survey at their first clinic visit (ie, prior to receiving care). Patients with language barriers, cognitive difficulties or atypical enrolment processes (eg, acute medical needs requiring emergency department referral) that preclude them from completing the survey are not eligible.

\section{Control patient characteristics for healthcare cost and utilisation analysis}

For the primary outcome analysis, patients in the control group will receive standard care at UofU Health, whether it is primary or specialty care. As part of the standard care, they might have a primary healthcare provider in addition to specialty providers who treat or help in managing their chronic health conditions.

To identify patients in the control group who have similar characteristics of the patients in the case group (ie, IOC patients) propensity score matching will be used to minimise observable confounders that could potentially affect the outcomes. We will match each IOC patient to two control subjects with propensity score matching (ie, nearest-neighbour matching) using logistic regression models. Matching will be based on variables such as: year of birth, sex, race, baseline Elixhauser Comorbidity
Index (or Charlson Comorbidity Index), insurance status (Medicaid), baseline numbers of inpatient, emergency room and outpatients visits, healthcare costs 1 year prior to index date and mental disorder status. Matching using callipers of a specified width will be used to match untreated subjects with a similar propensity score. Callipers of width 0.2 of the SD of the logit of the propensity score will be used because this width had estimates of intervention effect with lower mean squared error compared with other methods. If multiple control subjects have propensity score values that are equally close to that of the case subject, then one of these control subjects is selected at random. As we employ one-to-two matching without replacement, the final matched sample will consist of unique subjects in both groups. To assess the balance in the baseline covariates between the groups, the standardised difference methods for both continuous and categorical variables will be used. If standardised differences are $<10 \%$ (or 0.10 ), we assume that the imbalance between the groups is negligible. Entry into the study (ie, start of follow-up) of the IOC patients will be based on the first IOC visit date. Follow-up of the controls will begin the same dates as the matched IOC patients, providing equal follow-up time for both groups. The reason for one versus two matching is that it could improve small variance (ie, precision) without increase in bias. ${ }^{910}$

\section{Interventions (care at the IOC)}

When patients come to the IOC, they receive care from a multidisciplinary team consisting of physicians (including one physician consultant who specialises in Addiction Medicine), a nurse practitioner, a clinical pharmacist, registered nurse care manager, a licensed clinical social worker and medical assistants.

The physicians lead the care team, and are responsible for initial patient contact and recruitment, longitudinal acute and chronic disease management, preventive care services, interventions and referrals to specialty care, supported by the nurse practitioner. The clinical pharmacist (PharmD) regularly performs medication reconciliations, monitors potential adverse drug interactions, prescribes and manages selected chronic diseases (eg, diabetes, asthma, hypertension) using collaborative practice agreement, and promotes medication adherence. The pharmacist also performs ad hoc tasks as needed by the physician (eg, determining appropriate new medication therapies for patients, helping patients switch pharmacies or trouble-shooting problems with patients' diabetic equipment, if applicable). Further coaching is provided by medical assistants who will educate patients on disease management techniques to help them keep on track with their care plans. Because it has been recognised that the integration of physical and mental healthcare improves patient outcomes, a licensed clinical social worker integrates patients' mental health needs with their physical care plans. For patients who choose this option, they are able to receive short-term or long-term therapy services in the clinic. The Nurse Care Manager 
builds initial patient rapport through telephonic, electronic and home visits, and also connects patients to available community resources (eg, transportation). To help coordinate care between team members and patients, the Nurse Care Manager integrates and coordinates the interactions between IOC staff and patients, provides frequent 'touches' with the office through multiple channels, and coordinates internal and external appointment scheduling to facilitate timely and comprehensive care.

\section{OUTCOME MEASURES}

\section{Primary outcomes: healthcare costs and utilisation}

Costs

Healthcare costs of patients will be extracted from claims data and evaluated prospectively, from 24 months prior to IOC enrolment and up to 18 months after. Cost outcomes will include: total healthcare cost (sum of medical reimbursed amount and prescription reimbursed amount), inpatient cost, emergency department cost and prescription cost. Healthcare costs will be adjusted to 2018 dollars using Personal Healthcare Expenditure component of the National Health Expenditure Accounts ${ }^{11}$ for the cost calculations to reflect inflation over time. Both outcomes will be aggregated in each month for trends analysis and will be aggregated before and after the IOC intervention or index date (for the controls).

\section{Utilisation}

Patients' healthcare utilisation records will be collected from claims data and evaluated prospectively, from 24 months prior to IOC enrolment and up to 18 months after. Specific outcomes of interest include the number of hospital admissions, length of hospital stay, number of emergency department visits and office visits. This healthcare utilisation information will be identified from the place of services where patients receive healthcare services.

\section{Secondary outcomes: patient health and care experience/care quality}

Patient-reported outcomes of mental and physical health, and patients' experiences with their healthcare will be collected, as described below. Covariates will include patient background characteristics that may provide useful targets for intervention, or that may predict intervention efficacy.

\section{Patient health}

Health outcomes include general and specific measures of physical health, behavioural health and quality of life.

\section{Mental and behavioural health}

Includes the PROMIS depression, anxiety, sleep disturbance and applied cognitive general concerns scales. ${ }^{12}$ All surveys use a 5-point Likert-type scale with higher scores indicating higher severity. Other patient-reported outcomes include surveys of mania, ${ }^{13}$ psychosis, ${ }^{14}$ dissociation, ${ }^{14}$ personality disorder, ${ }^{14}$ repetitive thoughts ${ }^{15}$ and substance abuse. ${ }^{16}$

Physical health

Two self-rated health items will ask participants to indicate their general health status on a Likert-type scale (poor, fair, good, excellent) and visual analogue scale (from 0 to 100). The presence of physical symptoms will be assessed with the somatic severity scale. ${ }^{17}$

Quality of life

Quality of life indicators will be the PROMIS scales of pain interference, physical function and satisfaction with social roles and activities. ${ }^{12}$ Higher scores for the pain interference items reflected higher impact of pain on daily functioning, whereas higher scores for satisfaction with social roles or activities and physical function indicate increased role satisfaction and better physical functioning.

\section{Patient care experience/care quality}

Items on patients' experience with their healthcare, including trust in health providers and perceptions of provider communication, care coordination, access to care/community resources and self-efficacy for disease management.

\section{Access/referrals to community resources}

To determine patient access to community resources and referral by providers and clinic staff to community resources, participants will be asked three questions from the Patient Assessment of Chronic Illness Care (PACIC) survey. ${ }^{18}$ Patients will be asked, "Over the past 6 months, when receiving medical care, I was": (1) encouraged to attend programmes in the community that could help me; (2) asked how my work, family or social situation related to taking care of my illness and (3) helped to make plans for how to get support from my friends, family or community. The PACIC uses a 5-point Likert scale of almost never, generally not, sometimes, most of the time and almost always.

Trust

The Patient Trust Scale,$^{19}$ developed by Kao et al adapted from the Trust-in-Physicians scale, ${ }^{20}$ is a 10-question 5-point Likert scale, which asks questions concerning trust in one's physician on issues such as referrals, hospital admission, medical testing and medications.

\section{Communication}

The Modified Picker Survey, ${ }^{20}$ also modified by Kao et $a l$ from the Picker survey, ${ }^{21-23}$ is a seven-question survey using a 4-point scale (never, sometimes, usually, always) concerning patient and physician communication. Questions cover issues such as having enough time to explain reasons for the visit, if there was enough time for the physician to answer questions, and if patients were involved in decisions as much as they wanted to.

\section{Care coordination}

Three questions were selected from the continuity of care: when patients encounter several clinicians survey, ${ }^{24}$ 
which cover indicators of discontinuity, or where patients feel like their care is disjointed or feel abandoned by the healthcare system.

\section{Self-efficacy}

The self-efficacy to manage disease in general section from the self-efficacy scale ${ }^{25}$ will be used to measure patient motivation to manage their illness or disease. Questions cover topics such as knowing when to visit a doctor, managing emotional distress and patient confidence in managing their condition.

\section{Covariates: individual differences}

Covariates will include patient background characteristics that may provide useful targets for intervention, or that may predict intervention efficacy. Covariates will include items on patient demographics and other baseline characteristics, including health literacy, numeracy, acculturation and trait-like preferences for medical intervention (ie, medical maximising-minimising). Some covariates (ie, employment status and medical maximising-minimising preferences) may also be treated as time-varying or included as intervention outcomes in exploratory analyses.

\section{Demographics}

Participants will be asked five demographic questions which include age, gender, highest level of education completed, race and ethnicity.

\section{Health literacy}

To ascertain participant literacy, the Chew et al three-question health literacy screener will be used. ${ }^{26}$

\section{Numeracy}

The subjective numeracy scale is a 6-point Likert scale four-question survey developed by Fagerlin et $a l^{27} 28$ which asks patients their math skills without asking math questions.

\section{Employment status}

Participants will be asked four questions on their work situation. These questions are: 1) Do you work? 2) About how many hours do you work per week? 3) Does your health prevent you from working as many hours as you would like? and 4) If your health improved, how many hours per week would you like to work?

\section{Acculturation}

To understand patients' language (and social) preferences, the short acculturation scale for Hispanics ${ }^{29}$ was adapted to incorporate multiple languages. This survey uses a 5-point Likert scale. Question responses originally were 'only Spanish, Spanish better than English, both equally, English better than Spanish and only English'; or 'only Spanish, more Spanish than English, both equally, more English than Spanish and only English'. Instead of using 'Spanish', patients will be asked about their 'native language'. Only 4 questions out of the 12 question scale will be used. These questions are: 1) In general, what language(s) do you read and speak? 2) What language(s) do you usually speak at home? 3) In which language(s) do you usually think? and 4) What languages do you usually speak with your friends?

\section{Medical maximising-minimising preferences}

At all four assessments, participants are also asked to complete the medical maximiser-minimiser scale, ${ }^{30} \mathrm{a}$ 10-question survey that measures where patients fall along the spectrum of wanting to do as much as possible when it comes to their health (eg, aggressive treatments or tests of little diagnostic value) to as little as possible (eg, watchful waiting). Response options were on a 7-point Likert scale (strongly disagree to strongly agree). Examples of items included, 'It is important to treat disease even when it does not make a difference in survival' and "If I feel unhealthy, the first thing I do is to go to the doctor and get a prescription".

\section{Adverse Childhood Experiences}

At the baseline assessment, participants were asked to complete the Adverse Childhood Experiences (ACE) Study Questionnaire, ${ }^{31}$ a 17 -item survey that asks participants to indicate whether they have ever experienced a series of traumatic childhood experiences (eg, physical or sexual abuse, neglect) prior to 18 years of age $(0=$ no, $1=$ yes $)$.

\section{Timeline of survey administration}

An overview of all survey outcome measurements and timepoints are included in table 1. Participants in the study will be given surveys at four time-points over 18 months. Surveys will be administered at baseline and again at 6-month, 12-month and 18-month follow-ups. Shorter follow-up surveys will be administered every 6 months up to 18 months after baseline. The 6-month, 12-month and 18-month surveys will only include items on physical and mental/ behavioural health, quality of life, employment status, self-efficacy, medical maximising-minimising and quality of care (eg, trust, communication, care coordination, access/ referral to community resources and self-efficacy).

\section{Sample size}

Based on a sample size of $n=100$, we hypothesise that the mean difference in patients' reported health and care quality before and after IOC intervention will be up to $20 \%$. We assume the SD of the differences equals 0.10 and mean score at baseline $0.31 .^{32}$ Using these assumptions, we will obtain over $80 \%$ power to detect an effect size $=0.5$.

Statistical power for the healthcare cost was estimated based on an assumed SD (ie, US\$15 000) for change in the healthcare cost over 12 months of US $\$ 6139 .{ }^{33}$ The difference in healthcare costs between the case and the control groups is assumed to be $21 \% .{ }^{34}$ Considering these numbers and a 1:2 matching (100 cases to 200 controls), the minimum detectable treatment effect with $80 \%$ power, two-sided $\alpha=0.05$ is US $\$ 1222$ in the healthcare cost outcome. 
Table 1 Survey timeline

\begin{tabular}{|c|c|c|c|c|}
\hline & Baseline & 6 months & 12 months & 18 months \\
\hline \multicolumn{5}{|l|}{ Patient health } \\
\hline PROMIS applied cognition-general concerns & $x$ & $x$ & $\mathrm{x}$ & $\mathrm{x}$ \\
\hline PROMIS depression & $x$ & $x$ & $\mathrm{x}$ & $\mathrm{x}$ \\
\hline PROMIS pain interference & $x$ & $x$ & $\mathrm{x}$ & $\mathrm{x}$ \\
\hline PROMIS satisfaction with social roles and activities & $x$ & $x$ & $\mathrm{x}$ & $\mathrm{x}$ \\
\hline PROMIS sleep disturbance & $x$ & $x$ & $\mathrm{x}$ & $\mathrm{x}$ \\
\hline Mania & $\mathrm{x}$ & $\mathrm{x}$ & $\mathrm{x}$ & $x$ \\
\hline Somatic severity & $x$ & $x$ & $x$ & $x$ \\
\hline Psychosis & $x$ & $x$ & $x$ & $\mathrm{x}$ \\
\hline Dissociation & $x$ & $x$ & $x$ & $x$ \\
\hline Personality disorder & $x$ & $x$ & $x$ & $\mathrm{x}$ \\
\hline Substance use & $x$ & $x$ & $x$ & $x$ \\
\hline \multicolumn{5}{|l|}{ Care experience/quality } \\
\hline Access/referral to community resources & $x$ & $x$ & $x$ & $x$ \\
\hline Trust & $x$ & $x$ & $x$ & $x$ \\
\hline Communication & $x$ & $x$ & $x$ & $x$ \\
\hline Care coordination & $x$ & $x$ & $x$ & $x$ \\
\hline Self-efficacy & $x$ & $x$ & $\mathrm{x}$ & $\mathrm{x}$ \\
\hline Maximiser-minimiser & $x$ & $x$ & $x$ & $x$ \\
\hline Adverse childhood experiences & $x$ & & & \\
\hline
\end{tabular}

\section{Recruitment, enrolment and consent}

There are multiple recruitment processes for the study. All new IOC patients will be recruited at the time of their first visit to the IOC. To minimise bias, all patients at the IOC will be invited to enrol. Those who want to participate will enrol at that time. For all participants, informed consent will be completed through an electronic data capture when potential participants access the study survey link. Because surveys will be completed online, all participants will be informed that if they wish to participate, completion of the surveys will imply their consent.

\section{Data collection methods}

Healthcare costs/utilisation

The data for this study will be collected from claims databases for both the case and the control groups.

\section{Patient health/care quality}

Some data for this study will be collected by electronic data capture, and some data will be collected as part of routine care at the clinic for the intervention group. The survey is available to intervention participants through REDCap.

As this is a minimal risk study, data monitoring will be conducted by either the PI, a study coordinator or research nurse and/or a research assistant. Data will be monitored at least every 6 months to review and confirm participant eligibility and review missing data from survey responses. For participants who discontinue the study, data that were collected previously from completed surveys will still be included in the final analysis, using robust statistical methods to account for missing data and attrition. For participants who 
substantially deviate from the intervention protocol, the study staff will note this in a separate administrative form in the electronic data capture and their outcome data may be dropped from the final analysis (pending that they are a substantial outlier). Data regarding reasons for dropout will be collected (eg, death of a participant, relocation).

\section{DATA MANAGEMENT}

As all data are collected through electronic data capture, there will be no manual entry of survey data. Data will be stored on the secure electronic data capture server located at the UofU, as well as on password-protected computers and locked storage cabinets only accessible to the research team. All data which could link participants to their responses will be stored in a password-protected electronic folder separate from the survey responses and only accessible to the research team. Data will be monitored periodically (every 6 months) by the postdoctoral fellow to confirm patient eligibility, and to document missing data and participant dropout.

\section{STATISTICAL METHODS \\ Descriptive statistics}

Descriptive statistics such as mean, SD, frequency, proportion, and kernel density will be used to compare baseline characteristics (eg, comorbid conditions, mental disorder status, health utilisation) between IOC and control group patients for the primary analysis of healthcare costs/utilisation, and to identify potential outliers for both primary and secondary analyses. They will also be used to evaluate bivariate associations between predictors, covariates (eg, health literacy, numeracy and acculturation) and primary or secondary outcomes.

\section{Primary outcome measures: healthcare costs and utilisation} Healthcare costs/utilisation 24 months before and 18 months after intervention will be considered.

We will use parametric and non-parametric methods to compare costs between the two groups (ie, case and control groups). As the mean is the most useful statistic to evaluate costs related to the IOC intervention, we will calculate means and SD for total costs (ie, the sum of medical costs and medication costs) by group. We will explore cost data distributions graphically and statistically. With univariate and multivariate techniques, we will examine the relationship between intervention and total cost. As cost data are typically skewed, we will use non-parametric bootstrapping methods with 2000 pair-wise replications to compare mean costs and avoid distributional assumptions. CIs around the mean cost difference will be calculated with bias-corrected and accelerated methods. To examine healthcare costs, an estimated generalised linear regression with $\log$ link and gamma distribution will be applied. In order to examine healthcare utilisation, negative binomial regression will be used to handle overdispersion (ie, mean $\neq$ variance) of the number of inpatient or emergency room visits.

\section{Secondary outcome measures: patient health and care experience/quality}

The patient-reported health and quality of care outcomes include several domains with multiple questions. For ordinal or binary measures, conditional logistic regression will be used to predict the OR of a positive healthcare experience as a function of time in the IOC intervention (with first visit as the reference), after controlling for baseline characteristics such as patient demographics (eg, age, gender, employment status), comorbid conditions and healthcare utilisation. For these measures, a positive patient healthcare experience is defined as answering 'yes, somewhat' or 'yes, definitely' when there are three response categories (eg, 'no', 'yes, somewhat' and 'yes, definitely'), or answering 'yes' when there are two response categories ('yes' and 'no').

Longitudinal, multilevel regression analyses or growth models within an MLM framework will be used to assess patterns of change in continuous patient-reported outcomes (ie, self-efficacy, trust, care coordination) as a function of time in the intervention (again, with the first IOC visit as the reference time-point) across the four assessments at baseline, 6, 12 and 18 months. Analyses will be adjusted for baseline characteristics including age, gender, employment status, comorbid conditions and mental disorder status. Time-varying predictors will also be incorporated, such as employment status and chronic health conditions. In some cases, multivariate techniques may be used to combine patient-reported domains into a single outcome for subsequent modelling. For example, confirmatory factor analysis can be applied to load the indicators of trust in provider, communication, care coordination and access to community resources onto a single, underlying factor that reflects a patient's positive healthcare experience.

\section{Handling missing data}

Missing data are assumed to be in part at random and in part not at random. ${ }^{35}$ Full information maximum likelihood estimation methods will be applied that make use of all available data; this approach has been shown to reduce bias and increase efficiency in the estimation of model parameters and their SEs, relative to traditional missing data approaches. ${ }^{36}$ Longitudinal attrition analyses will help to identify missing data patterns and mechanisms of missingness (eg, the extent to which patient demographics and health variables predict missingness across the 18 months of follow-up).

\section{ETHICS AND DISSEMINATION}

This study of health and healthcare improvements among the highest-cost, highest-need patients in the UofU Health 
system, most of whom are publicly insured and have both medical and behavioural health issues, requires special consideration of barriers related to culture, language, health literacy and social determinants (eg, homelessness, unemployment, lack of social support). Any modifications to the study will be submitted to the UofU institutional review board (IRB) for approval.

Dissemination of results from this study will occur locally and nationally. Specifically, primary outcomes from the healthcare utilisation and cost analysis will be shared in meetings with the health plan/medical group and with other university clinics. Manuscripts based on the intervention care model, as well as primary and secondary outcomes at 6,12 and 18 months of follow-up will be submitted to scientific, peer-reviewed journals in the field. Results will also be presented nationally at scientific meetings.

\section{Access to data}

Only members of the study team will have access to participant surveys and identifying information (PHI). All PHI collected in the study will be stored in a password-protected environment at the UofU. This information will not be shared outside of the study team and the UofU IRB, unless IRB approval has been obtained. There will be no limits to access of the data for members of the research team. Once project data collection is complete, investigators will be able to access the dataset to conduct additional analyses after appropriate IRB approvals have been obtained.

\section{DISCUSSION}

A small percentage of individuals account for a disproportionately large amount of US health spending, placing an appreciable burden on the healthcare system and the economy. A subset of these patients are medically complex, and they typically have challenging social circumstances and unmet behavioural health concerns, which exacerbate their health problems and drive their acute and costly utilisation. Although intensive outpatient programmes (also referred to as 'super-utiliser programmes' or 'ambulatory intensive care units') are being developed across the country to respond to the need for innovative care models for these high-need, high-risk patients, these programmes must be tailored to the communities they serve. The IOC was developed at the UofU Health, which aims to deliver comprehensive, coordinated and tailored care to the highest-need and costliest patients. This prospective, comparative case-control study aims to evaluate the effectiveness of the IOC in regard to reductions in cost and utilisation, as well as the IOC's efficacy at improving patient-reported outcomes that reflect patients' health, care experience and engagement in self-care and disease management.

Some possible limitations of the study include its prepost and quasi-experimental design, which may limit inferences of causality relative to randomised controlled trials. Also, difficulty with patient recruitment, commonly noted in similar evaluation studies, might limit the sample size and statistical power for testing the study hypotheses. Lastly, our study design will not allow us to specify which specific components of the IOC are the most effective at improving patient outcomes or at reducing costs and utilisation. Examining the efficacy of the IOC in its entirety will be a crucial first step in evaluation, which can be used to guide future research efforts that aim to identify the elements that help to explain the intervention's impact on outcomes of interest. Despite these common challenges, however, the results from this study will be used to inform ways to improve the implementation of the IOC to provide better care for patients, leading to longterm improvements in population health and cost-effectiveness. The IOC's care model will also provide insight and guide the development of high-risk/high-need programmes nationwide that aim to reduce the fragmentation of care and improve clinical outcomes among their communities' highest-utilising patients.

\section{Author affiliations}

${ }^{1}$ Department of Population Health Sciences, University of Utah School of Medicine, Salt Lake City, Utah, USA

${ }^{2}$ VA Center for Informatics Decision Enhancement and Surveillance (IDEAS), Salt Lake City, Utah, USA

${ }^{3}$ Department of Health and Kinesiology, University of Utah, Salt Lake City, Utah, USA ${ }^{4}$ Department of Family and Preventive Medicine, University of Utah School of Medicine, Salt Lake City, Utah, USA

${ }^{5}$ Department of Pediatrics, University of Utah School of Medicine, Salt Lake City, Utah, USA

Acknowledgements The authors would like to thank members of the IOC project team that contributed to this work: Craig Holbrook, Ryan Morley, Paige Patterson, Lara Newbold and Anna Cassell.

Contributors EMO, AF, PW conceptualised the study and its design. JK provided statistical expertise. BK provides the medical care. All authors contributed to the write-up of the study protocol by providing comments on drafts written by BLB and $M L$ and approved the final manuscript.

Funding This work is supported by the University of Utah Medical Group (UUMG). Funding for the evaluation was also received by the Hearst Foundations.

Competing interests None declared.

Patient consent Not required.

Ethics approval Ethics approval has been granted for this study by the University of Utah Health Sciences Institutional Review Board (approval number IRB_00100959).

Provenance and peer review Not commissioned; externally peer reviewed.

Open access This is an open access article distributed in accordance with the Creative Commons Attribution Non Commercial (CC BY-NC 4.0) license, which permits others to distribute, remix, adapt, build upon this work non-commercially, and license their derivative works on different terms, provided the original work is properly cited, appropriate credit is given, any changes made indicated, and the use is non-commercial. See: http://creativecommons.org/licenses/by-nc/4.0/.

\section{REFERENCES}

1. Conwell LJ, Cohen JW. Characteristics of persons with high medical expenditures in the u.S. Civilian noninstitutionalized population, 2002. Meps: Medical expenditure paney survey statistical Brief \#73. 2005 https://meps.ahrq.gov/data_files/publications/st73/stat73.pdf.

2. Cohen SB. The concentration and persistence in the level of health expenditures over time: estimates for the U.S. population, 20112012. MEPS: medical expenditure paney survey statistical brief \#449. 
2014 http://www.meps.ahrq.gov/mepsweb/data_files/publications/ st449/stat449.pdf.

3. Stanton MW, Rutherford MK. The High Concentration of U. S. Health Care Expenditures. Rockville 2005.

4. Thomas-Henkel C, Hendricks T, Church K. Opportunities to Improve Models of Care for People with Complex Needs: Literature Review, 2015.

5. Long P, Abrams M, Milstein A, et al. Effective Care for High-Need Patients. Washington, D.C, 2017.

6. Warning W, Wood J, Letcher A, et al. Working with the superutilizer population: The experience and recommendations of five pennsylvania programs. 2014 http://www.aligning4healthpa.org/pdf/ High_Utilizer_Report.pdf.

7. Greene J, Hibbard JH, Sacks R, et al. When patient activation levels change, health outcomes and costs change, too. Health Aff 2015;34:431-7.

8. Hibbard JH, Greene J, Shi Y, et al. Taking the long view: how well do patient activation scores predict outcomes four years later? Med Care Res Rev 2015;72:324-37.

9. Austin PC. Statistical criteria for selecting the optimal number of untreated subjects matched to each treated subject when using many-to-one matching on the propensity score. Am J Epidemiol 2010;172:1092-7.

10. Rassen JA, Shelat AA, Myers J, et al. One-to-many propensity score matching in cohort studies. Pharmacoepidemiol Drug Saf 2012;21 Suppl 2(S2):69-80.

11. Agency for Healthcare Research and Quality. Using appropriate price indices for analysis of health care expenditures or income across multiple years. Medical Expenditure Panel Survey. https://meps.ahrq gov/about_meps/Price_Index.shtml. Published. 2017. (Accessed 20 Aug 2002).

12. Cella D, Yount S, Rothrock N, et al. The Patient-Reported Outcomes Measurement Information System (PROMIS). Med Care 2007;45(Suppl 1):S3-S11.

13. Altman EG, Hedeker D, Peterson JL, et al. The Altman Self-Rating Mania Scale. Biol Psychiatry 1997;42:948-55.

14. American Psychiatric Association. DSM-5 Self-Rated Leve 1 Cross-Cutting Symptom Measure-Adult: In. Diagnostic and Statistical Manual of Mental Disorders. 5th Edition. Washington: D.C: American Psychiatric Association, 2013. https://www.psychiatry.org/psychiatrists/practice/dsm/ educational-resources/assessment-measures.

15. Goodman WK. LEVEL 2-Repetitive Thoughts and Behaviors-Adult (Adapted from the Florida Obsessive-Compulsive Inventory [FOCI] Severity Score [Part B], 1994.

16. National Institute on Alcohol Abuse and Alcoholism. National Epidemiologic Survey on Alcohol and Related Conditions-III (NESARC-III). Rockville, MD: National Institutes of Health.

17. Kroenke K, Spitzer RL, Williams JB. The PHQ-15: validity of a new measure for evaluating the severity of somatic symptoms. Psychosom Med 2002;64:258-66.

18. Glasgow RE, Wagner EH, Schaefer J, et al. Development and validation of the Patient Assessment of Chronic Illness Care (PACIC). Med Care 2005;43:436-44.
19. Kao AC, Green DC, Zaslavsky AM, et al. The relationship between method of physician payment and patient trust. JAMA 1998;280:1708-14

20. Anderson LA, Dedrick RF. Development of the trust in physician scale: A measure to assess interpersonal trust in patient-physician relationships. Psychol Rep 1990;67:1091-100.

21. Cleary PD, Edgman-Levitan S, Roberts M, et al. Patients evaluate their hospital care: A national survey. Health Aff 1991;10:254-67.

22. Edgman-Levitan S, Cleary PD. What information do consumers want and need? Health Aff 1996;15:42-56.

23. Cleary PD, Edgman-Levitan S, McMullen W, et al. The relationship between reported problems and patient summary evaluations of hospital care. QRB Qual Rev Bull 1992;18:53-9.

24. Haggerty JL, Roberge D, Freeman GK, et al. Validation of a generic measure of continuity of care: when patients encounter several clinicians. Ann Fam Med 2012;10:443-51.

25. Lorig K, Stewart A, Ritter P, et al. Outcome Measures for Health Education and Other Health Care Interventions. Thousand Oaks, CA: Sage Publications, Inc, 1996.

26. Chew LD, Bradley KA, Boyko EJ. Brief questions to identify patients with inadequate health literacy. Fam Med 2004;36:588-94.

27. Fagerlin A, Zikmund-Fisher BJ, Ubel PA, et al. Measuring numeracy without a math test: development of the Subjective Numeracy Scale. Med Decis Making 2007;27:672-80

28. Zikmund-Fisher BJ, Smith DM, Ubel PA, et al. Validation of the subjective numeracy scale: Effects of low numeracy on comprehension of risk communications and utility elicitations. Med Decis Making 2007;27:663-71.

29. Marin G, Sabogal F, Marin BV, et al. Development of a short acculturation scale for hispanics. Hisp J Behav Sci 1987;9:183-205.

30. Scherer LD, Caverly TJ, Burke J, et al. Development of the medical maximizer-minimizer scale. Health Psychol 2016;35:1276-87.

31. Felitti VJ, Anda RF, Nordenberg D, et al. Relationship of childhood abuse and household dysfunction to many of the leading causes of death in adults. The Adverse Childhood Experiences (ACE) Study. Am J Prev Med 1998;14:245-58.

32. Carlin CS, Christianson JB, Keenan P, et al. Chronic illness and patient satisfaction. Health Serv Res 2012;47:2250-72.

33. Zulman DM, Pal Chee C, Ezeji-Okoye SC, et al. Effect of an intensive outpatient program to augment primary care for high-need veterans affairs patients: A randomized clinical trial. JAMA Intern Med 2017;177:166.

34. Stremikis K, Connors C, Hoo E. Intensive outpatient care program: A care model for the medically complex piloted by employers. Commonw Fund 2017. http://www.commonwealthfund.org/ / media/files/publications/case-study/2017/sep/stremikis_intensive_ outpatient_care_prog_cs.pdf.

35. Graham JW. Missing data analysis: making it work in the real world. Annu Rev Psychol 2009:60:549-76.

36. Enders $C$, Bandalos D. The relative performance of full information maximum likelihood estimation for missing data in structural equation models. Structural Equation Modeling: A Multidisciplinary Journal 2001;8:430-57. 